\title{
Appreciation to Empirical Software Engineering reviewers of 2016
}

For helping us deliver timely decisions to our authors, the Editors-in-Chief and Publisher would like to thank the following individuals that contributed reviews between November 1 , 2015 and November 1, 2016. We applaud your efforts and dedication to the community.

Silvia Abrahao

Alain Abran

Rui Abreu

Bram Adams

Emil Alégroth

Nasir Ali

Raian Ali

Mohamed Almorsy

Vander Alves

Lefteris Angelis

Mauricio Aniche

Giulio Antoniol

John Anvik

Venera Arnaoudova

Saïd Assar

George Baah

Alberto Bacchelli

Doo-Hwan Bae

Ebrahim Bagheri

Teresa Baldassarre

Paulo Barbosa

Amid Khatibi Bardsiri

Earl Barr

Gabriele Bavota

Saman Bazrafshan

Sarah Beecham

Andrew Begel

Jonathan Bell
Moritz Beller

David Benavides

Ayse Bener

Beatriz Bernardez

Richard Berntsson-Svensson

Daniel Berry

Stefanie Betz

Cor-Paul Bezemer

Christian Bird

Tegawende Bissyande

Elizabeth Bjarnason

Kelly Blincoe

Markus Borg

Amiangshu Bosu

David Bowes

Marco Brambilla

Olwen Brereton

David Budgen

Simon Butler

Jordi Cabot

Haipeng Cai

Fabio Calefato

Javier Canovas Izquierdo

Giovanni Cantone

Rafael Capilla

Andrea Capiluppi

Josep Carmona

Jeffrey Carver 
Mariano Ceccato

Humberto Cervantes

W.K. Chan

Michel Chaudron

Peter Chen

James Clause

Jane Cleland-Huang

Michael Coblenz

Thomas Connolly

Steve Counsell

Gianpaolo Cugola

Aldo Dagnino

M. Daneva

Andrea De Lucia

Thomas Debeauvais

Rob DeLine

Serge Demeyer

Stefano Di Alesio

Massimiliano Di Penta

Davide Di Ruscio

Stephan Diehl

Oscar Dieste

Junhua Ding

Torgeir Dingsøyr

Bogdan Dit

Robert Dyer

Neil Ernst

Sandra Fabbri

Davide Falessi

John Favaro

Dror Feitelson

Michael Felderer

Rudolf Ferenc

Eduardo B. Fernandez

Eduardo Figueiredo

Vladimir Filkov

Patrick Francis

Harald Gall

Alessio Gambi

Cigdem Gencel

Daniel German

Sepideh Ghanavati

Asif Gill

Dennis Goldenson

Leah Goldin

Cristina Gómez

Tony Gorschek
Georgios Gousios

Carmine Gravino

Daniel Graziotin

Mark Grechanik

Christiane Gresse von Wangenheim

Michael Grottke

Yann-Gael Gueheneuc

Sonia Haiduc

Tracy Hall

Stefan Hanenberg

Dan Hao

Mark Harman

Rachel Harrison

Ahmed Hassan

Safwat Hassan

Edgar Hassler

Robert Heinrich

Andrea Herrmann

Yoshiki Higo

Emily Hill

Abram Hindle

Andre Hora

B.M. Mainul Hossain

Robert Hughes

Akinori Ihara

Zohaib Iqbal

Juha Itkonen

Clem Izurieta

Letizia Jaccheri

Dietmar Jannach

Slinger Jansen

Ross Jeffery

Yue Jia

Yan Jiachen

Bo Jiang

Lingxiao Jiang

Zhen Ming (Jack) Jiang

Natalia Juristo

Jan Jürjens

Huzefa Kagdi

Yasutaka Kamei

Rick Kazman

Diane Kelly

Marouane Kessentini

Jacky Keung

Foutse Khomh

Dongsun Kim 
Miryung Kim

Sunghun Kim

Barbara A. Kitchenham

Eric Knauss

Pavneet Singh Kochhar

Fábio Kon

Jyrki Kontio

Anne Koziolek

Heiko Koziolek

Nicholas Kraft

Chandra Krintz

Ivo Krka

Srdan Krstic

P Kruchten

Casper Lassenius

Thomas LaToza

Kim Lauenroth

Luigi Lavazza

Niklas Lavesson

Julia Lawall

Lucas Layman

Philipp Leitner

Maurizio Leotta

Timothy Lethbridge

Grace Lewis

Mario Linares-Vásquez

David Lo

Christopher Lokan

Cristina Lopes

Roberto Lopez-Herrejon

Walid Maalej

Anas Mahmoud

Ivano Malavolta

Jonathan Maletic

Haroon Malik

Vladimir Mandic

Senthil Mani

Mika Mäntylä

Darko Marinov

Salvador Martínez

Katsuhisa Maruyama

Wes Masri

Santiago Matalonga

Nicholas Matragkas

Kenichi Matsumoto

Fergal McCaffery

John McGregor
Shane McIntosh

Collin McMillan

Ernestina Menasalvas

Emilia Mendes

Daniel Méndez Fernández

Tim Menzies

Marjan Mernik

Leandro Minku

Mehdi Mirakhorli

Andrea Mocci

Audris Mockus

Nils Moe

Parastoo Mohagheghi

Martin Monperrus

Sandro Morasca

Laura Moreno

Luca Mottola

Henry Muccini

Pradeep Murukannaiah

Sarah Nadi

Meiyappan Nagappan

Jaechang Nam

Roberto Natella

Jerzy Nawrocki

Iulian Neamtiu

Shiva Nejati

Hoan Nguyen

Tien Nguyen

William Nichols

Nan Niu

Markku Oivo

Rocco Oliveto

Barbara Paech

Richard Paige

Fabio Palomba

Annibale Panichella

Sebastiano Panichella

Victor Pankratius

Chris Parnin

Karthik Pattabiraman

Cecile Peraire

Fayola Peters

Kai Petersen

Justyna Petke

Dietmar Pfahl

Fiona Polack

Macario Polo Usaola 
Peter Popov

Denys Poshyvanyk

Pasqualina Potena

Simon Poulding

Michael Pradel

Rafael Prikladnicki

Giuseppe Procaccianti

Fabio Queda Silva

Lukasz Radlinski

R. Ramler

Ayushi Rastogi

Baishakhi Ray

Patrick Rempel

Filippo Ricca

Peter Rigby

Romain Robbes

Gregorio Robles

Pilar Rodríguez

Marc Roper

Rasmus Ros

Louis Rose

Chanchal Roy

Barbara Russo

Mehrdad Sabetzadeh

Ripon Saha

Houari Sahraoui

Hitesh Sajnani

Dina Salah

Norsaremah Salleh

Federica Sarro

Abdel Salan Sayyad

Giuseppe Scanniello

Klaus Schmid

Sergio Segura

Christoph Seidl

Bran Selic

Alexander Serebrenik

Weiyi Shang

Bonita Sharif

David Shepherd

Emad Shihab

Alberto Sillitti

Jouni Similä

Guttorm Sindre

Harvey Siy

Darja Smite

Will Snipes
Martin Solari

Jerffeson Souza

Diomidis Spinellis

Praveen Srivastava

Mark Staples

Miroslaw Staron

Igor Steinmacher

Robert Stoddard

Klaas-Jan Stol

Katie Stolee

Viktoria Stray

Chengnian Sun

Alistair Sutcliffe

Mark Syer

Damian Tamburri

Lin Tan

Antony Tang

Yida Tao

Ewan Tempero

Thomas Thuem

Suresh Thummalapenta

Jeff Tian

Inger Anne Tondel

Roberto Tonelli

Marco Torchiano

Ayse Tosun

Christoph Treude

Nikolaos Tsantalis

Jason Tsay

Michael Unterkalmsteiner

Marco Tulio Valente

Juan Vara

Bogdan Vasilescu

Sira Vegas

Christopher Vendome

Stefan Wagner

James Walden

Gursimran Walia

Robert Walker

Neil Walkinshaw

Haoyu Wang

Shaowei Wang

Heinz Roland Weistroffer

Jim Whitehead

Halfond William

Manuel Wimmer

Krzysztof Wnuk 
Katinka Wolter

Xin Xia

Min Xie

Wei Xu

Aiko Yamashita

Ye Yang

Annie Ying

Koen Yskout

Tingting Yu

Yijun Yu

Apostolos Zarras

Hongyu Zhang

Lingming Zhang

Pengcheng Zhang

Yiji Zhang

Yuanyuan Zhang

Yunhui Zheng

Ying Zou 